We present an unusual case of sepsis in a 23 year old lady of 14 weeks gestation (para $1+1$ ) who presented with epigatric pain. Initial presentation was with bilious vomiting which became blood stained. She reported not opening bowels for 2 days however had passed flatus. There was no significant past medical history with only appendicectomy performed 5 years previously. On examination abdomen was slightly distended with tenderness in the epigastric region. Bloods were: WCC 2.5, Neut 1.5. Other bloods including amylase were normal. She was reviewed by the surgical team 'biliary colic' and an abdominal ultrasound performed, was normal. She was reviewed by medical, surgical, obstetric and gastroenterology teams and finally the diagnosis was of hyperemesis gravidarum.

She was readmitted 48 hours later with pyrexia, tachycardia and hypotension. An ultrasound was performed which demonstrated increased free fluid and an emergency laparotomy was performed. Findings were that of a perforated distal ileum. A resection of the ascending colon and terminal ileum was performed with an ileostomy. Unfortunately, 3 days post operatively she miscarried despite the fetal heart being present immediately post operatively.

During recovery she admitted that her sister in law had contracted tuberculosis and she herself had not been vaccinated. Investigations for $\mathrm{TB}$ are currently ongoing. Intra-abdominal fluid samples have confirmed the presence of ESBL.

Although an unusual presentation, this case highlights the importance of maintaining a high suspicion of sepsis. This is especially true where blood results suggest this, in the absence of other features and ongoing symptoms.

\section{PM.89 SEVERE HEADACHES IN PREGNANCY. FIRST PRESENTATION OF ARNOLD CHIARI MALFORMATION TYPE 1}

doi:10.1136/archdischild-2013-303966.170

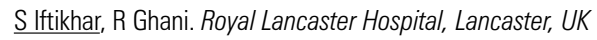

Arnold Chiari malformation is a malformation of the brain which consists of a downward displacement of the cerebellar tonsils through the foramen magnum. Type 1 Arnold Chiari is generally asymptomatic during childhood. It can typically present in women during early adulthood and can be a cause of unexplained headaches and cerebellar symptoms.

We present a case of 32 year old primigravida with severe headache and visual loss for the first time in pregnancy. She had a twin pregnancy after successful IVF. She also developed gestational diabetes. Magnetic resonance imaging of the brain showed Arnold Chiari malformation type 1 . We describe the management of this case during the antenatal and intrapartum period. She was delivered by emergency caesarean section under general anaesthetic.

Her visual loss was thought to be secondary to optic neuropathy with an unknown cause and it remains a clinical dilemma until now.

We discuss multidisciplinary team management and careful anaesthetic assessment in such cases.

\section{PM.90 INCIDENTAL PHAEOCHROMOCYTOMA IN PREGNANCY}

doi:10.1136/archdischild-2013-303966.171

BF Chen, S Al-Samarrai, M Rathi, J Rajeswary. King's Mill Hosptial, Sutton-inAshfield, UK

Introduction Phaeochromocytoma, a catecholamine-producing endocrine tumour, is a life-threatening condition to the mother and fetus. The incidence of phaeochromocytoma in pregnancy is extremely rare, 1 in 54000 pregnancies $^{1}$. If it remains undiagnosed and untreated, maternal and fetal mortality amounts to $40-50 \%^{23}$. Classically, physicians search for the tumour in hypertensive patients with paroxysmal symptoms such as headache, sweating or palpitations. However, our patient presented atypically and would have been left undiagnosed.

Case presentation A 21-year-old presented after a fall at 24 weeks gestation, with loin pain on the contralateral side to the fall. This prompted an ultrasound scan that demonstrated a $9 \mathrm{~cm}$ 'haematoma' above the right kidney. As she was claustrophobic, she declined magnetic resonance imaging (MRI). A repeat ultrasound was performed which showed the mass to be unchanged. Suspicion arose and an MRI under sedation was performed (as recommended to look for adrenal/renal mass in pregnancy ${ }^{4}$ ). This confirmed an $8 \mathrm{~cm} \times 7 \mathrm{~cm}$ mass superior to the right kidney. An endocrinologist reviewed her and serum and urine biochemistry investigations were performed.

Her urinary 3-methoxytyramine and serum dopamine levels were raised ${ }^{6}{ }^{2}$ indicating possible phaechromocytoma. She did not need $\alpha$-adrenoceptor blockade, as she remained asymptomatic throughout her pregnancy. She was referred to a tertiary hospital where an elective Caesarean section and surgical tumour removal were performed simultaneously.

Conclusion Diagnosing phaeochromocytoma in an asymptomatic pregnant patient is challenging. However, when diagnosed, a multidisciplinary team approach (obstetrician, surgeon and endocrinologist) is vital in the management of this rare disorder.

\section{REFERENCES}

1. Lenders JWM. Phaeochromocytoma and pregnancy: a deceptive connexion European Journal of Endocrinology 2012;161:143-150.

2. Schenker JG \& Chowers I. Phaeochromocytoma and pregnancy. Review of 89 cases. Obstetrical and Gynecological Survey 1971;26:739-747.

3. Dean RE. Phaeochromocytoma and pregnancy. Obstetrics and Gynecology 1958;11: $35-42$

4. Lenders JW, Eisenhofer G, Mannelli M, et al. Phaeochromocytoma. Lancet 2005;366:665-675

5. Peleg D, Munsick RA, Diker D, Goldman JA \& Ben-Jonathan N. Distribution of catecholamines between fetal and maternal compartments during human pregnancy with emphasis on L-dopa and dopamine. Journal of Clinical Endocrinology and Metabolism 1986:62:911-914.

6. Lenders JW, Pacak K, Walther MM, Linehan WM, Mannelli M, Friberg P, Keiser HR, Goldstein DS \& Eisenhofer G. Biochemical diagnosis of phaeochromocytoma: which test is best? Journal of the American Medical Association 2002;287: 1427-1434.

\section{PM.91 HAEMATOLOGICAL INDICES IN PREGNANCY: AN IRISH TERTIARY CENTRE EXPERIENCE}

doi:10.1136/archdischild-2013-303966.172

${ }^{1} \mathrm{CM}$ McCarthy, ${ }^{2} \mathrm{MR}$ Cahill, ${ }^{1} \mathrm{~K}$ O'Donoghue. ${ }^{1}$ Department of Obstetrics and Gynaecology, Cork University Maternity Hospital, Cork, Ireland; '2Department of Haematology, Cork University Hospital, Cork, Ireland

Many haematological changes occur during pregnancy to accommodate maternal and fetal needs. Thus, monitoring of this patient groups' haematological indices are imperative. International guidelines recommend minimum haematological sampling at booking and 28 weeks.

A prospective audit was conducted between January and April 2012. Postnatal patient charts were randomly sampled, and relevant data extracted. This was entered into a secure database. Haematological indices from throughout pregnancy and within 1 week of the postnatal period were extracted from institutional laboratory systems retrospectively.

176 patients were included in our sample group, with a total of 757 samples taken. The average age of patients was 31 . Within this sample, there were 100 vaginal deliveries and 76 caesarean 
deliveries, with 3 post-partum haemorrhages. Birth weights ranged from $2.37 \mathrm{~kg}$ to $4.9 \mathrm{~kg}$. 155 patients had more than 2 sets of haematological investigations during pregnancy, with 83 patients having more than 5 measurements. $39.7 \%$ had haematinic investigations performed, with 48 of these patients had suboptimal ferritin levels. Only 3 patients were defined as anaemic before 12 weeks gestation.

There are currently no national guidelines regarding appropriate haematological investigations in pregnancy. This study demonstrates the demographics and frequency of haematological investigation in our population. We have also identified the proportion of anaemia demonstrated at varying gestations.

\section{PM.92 FACILITATION OF VAGINAL DELIVERY IN AN INFANT WITH COMPLETE HEART BLOCK (CHB) SECONDARY TO MATERNAL ANTI RO ANTIBODIES}

doi:10.1136/archdischild-2013-303966.173

L Tripathi, S Shebani, I Bruce, L Byrd. University hospital of central Manchester, St Mary's Hospital and MRI, Manchester, UK

A 36-year-old, G4P4 presented at 7 weeks gestation, with known Sjorgen's Syndrome (Lupus anticoagulant and Anti Ro antibody positive) and previous deep vein thrombosis. At 20 weeks gestation both anomaly and fetal echocardiogram were undertaken and confirmed as normal. However 10 days later repeat echocardiogram confirmed congenital heart block $(\mathrm{CHB})$, ventricular rate $60 \mathrm{bpm}$. There was no evidence of either fetal hydrops or heart failure. The couple was counselled regarding the potentially poor outcome.

The pregnancy continued; the fetus remained healthy albeit with CHB. Options for delivery were regularly discussed. Given both her parity and history of quick labours she was keen, if possible, to avoid surgery. Serial growth scans confirmed good growth velocity. Comprehensive evaluation of heart function and fetal circulation (CVP score) and fetal well being (Biophysical Score) was assessed and shown to be maintained throughout pregnancy and at term when she presented in early labour. Labour progressed rapidly to delivery of a female infant, in excellent condition (Apgars of 9 at 1 and 5 minutes), birth weight $3.02 \mathrm{~kg}$. The newborn was transferred to SCBU and an echocardiogram re affirmed $\mathrm{CHB}$ with good cardiac function. She established demand feeding over the next $48-72$ hours without compromise and was discharged home with mum within the week. She remains well and has not yet required pacing (age 1 year).

Discussion The strategy of close fetal surveillance using both biophysical profile (BPP) and cardiovascular profile (CVP) scoring during both pregnancy and early labour facilitated a spontaneous vaginal delivery at term.(1)

\section{REFERENCE}

1. Huhta JC. Right ventricular function in the human fetus. J Perinat Med 2001; 29:381-9.

\section{PM.93 A COMPLICATED CASE OF GIANT VULVAL CONDYLOMATA IN PREGNANCY}

doi:10.1136/archdischild-2013-303966.174

S McGowan, J McManus, S McNeil. Altnage/vin Area Hospital, Western Health and Social Care Trust, Northern Ireland, Londonderry, UK

Anogenital warts are one of the most common sexually transmitted diseases in the UK caused by HPV infection. ${ }^{12}$ The condition is not purely benign with significant symptoms present in some patients related to severity and site of disease with an increased risk of cervical and other anogenital cancers. ${ }^{2}{ }^{3} \mathrm{HPV}$ infection is higher in pregnant women with rapid growth of warts observed which is thought to be related to immunological factors. ${ }^{4}$ Treatment, even in non-pregnant individuals is difficult and cure elusive. ${ }^{5}$ Treatment options are limited in pregnancy to conservative, non-teratogenic medical management and surgery. ${ }^{45}$ We present a case of massive condylomata in a pregnant patient complicated by brittle diabetes and mental health issues.

A 30 year old parous patient presented in early pregnancy with hyperemesis, poorly controlled type1 diabetes and a massive growth of vulval warts causing severe pain and difficulty mobilising. This large exophytic mass covered the vulvae and would preclude vaginal delivery. Genito-urinary medicine suggested a conservative course of treatment. Subsequently the patient had several admissions with hyperemesis and poor glycaemic control. The patient disclosed suicidal ideation related to the vulval warts and desire to not continue pregnancy. A termination of pregnancy was requested on this basis. Psychiatry review documented a depressive episode and suicide risk. In view of these factors a surgical resection was performed. This was complicated by significant blood loss requiring return to theatre, blood transfusion and HDU admission. Currently the pregnancy is ongoing.

\section{REFERENCES}

1. Alder MW. Genital warts and molluscum contagiosum. British Medical Journal 1984;288:213-15.

2. Gall SA. Female genital warts: global trends and treatments. Infectious Diseases in Obstetrics and Gynecology 2001:9:149-54.

3. Breen D, Bleday R. Condylomata acuminata (Anogenital warts). UpToDate 2012, www.uptodate.com/contents/condylomata-acuminata-anogenital-warts (accessed October 2012).

4. Gearhart P, et al. Human Papillomavirus Treatment \& Management, Medscape, http:// emedicine.medscape.com/article/219110-overview (accessed October 2012).

5. Carusi D. Treatment of vulvar and vaginal warts, UpToDate 2012, www.uptodate.com/contents/treatment-of-vulvar-and-vaginal-warts laccessed October 2012).

\section{PM.94 THE MANAGEMENT OF A TWIN PREGNANCY IN A PATIENT WITH MAGIC SYNDROME}

doi:10.1136/archdischild-2013-303966.175

A Fabre-Gray, D Mahendran. Gloucestershire Royal NHS Foundation Trust, Gloucester, UK

In 1985 Firestein et al, first described patients with co-existent relapsing polychondritis and Behçet's Disease. They proposed the acronym MAGIC syndrome (Mouth And Genital ulcers with Inflamed Cartilage).

This is a rare condition with no more than twenty cases described in the literature to date.

This case is the first to report the management of a pregnancy in a patient with MAGIC syndrome.

The autoimmune pathogenesis can potentially damage cartilage anywhere in the body, including cardiac valves and tracheal cartilage. Therefore a multidisciplinary approach to the management of this pregnancy was paramount.

We describe her antenatal, intrapartum and post-partum management specific to this condition and demonstrate how this led to a safe delivery of her twins.

\section{PM.95 SYSTEMIC LUPUS ERYTHEMATOSUS, IMMUNOSUPPRESSION AND SOUAMOUS CELL CARCINOMA OF THE TONGUE DURING PREGNANCY}

doi:10.1136/archdischild-2013-303966.176

${ }^{1}$ JD Unsworth, ${ }^{2} \mathrm{~A}$ Baldwin, ${ }^{3} \mathrm{~L}$ Byrd. ${ }^{1}$ Fairfield General Hospital, Manchester, UK; ${ }^{2}$ North Manchester General Hospital, Manchester, UK; ${ }^{3}$ St Mary's Hospital, Manchester, UK

This case describes a 29 year old cigarette smoker with underlying Systemic Lupus Erythematosus (SLE) treated aggressively with 